\title{
Impulse Amplification in a Traveling-Wave Tube-I: Simulation and Experimental Validation
}

\author{
Mark C. Converse, Member, IEEE, John H. Booske, Senior Member, IEEE, and Susan C. Hagness, Member, IEEE
}

\begin{abstract}
This paper presents a new one-dimensional timedomain model to be used in the analysis of the pulse response of the helix traveling-wave tube (TWT). It incorporates a novel method of implementing the effects of waveguide dispersion in one dimension. This new model has been successfully compared to an established frequency-domain model, and both time- and frequency-domain models have been compared to the impulse response of an experimental TWT. This comparison illustrates the efficacy of this new time-domain model.
\end{abstract}

Index Terms-Electron trapping, impulse amplification, impulse radar, impulse radio, microwave amplifier, time domain, traveling-wave tube (TWT), ultrawideband (UWB).

\section{INTRODUCTION}

$\mathbf{R}$ ESEARCH into traveling-wave tubes (TWTs) has been going on since the 1940 s, when the beneficial interaction between an electron beam and an electromagnetic wave guided by a helical waveguide was demonstrated [1]. During that time, numerous experiments have been performed and nonlinear models [2]-[5] have been developed. These models range from one-dimensional (1-D) [2] to three-dimensional (3-D) [6], [7]. Some have seen significant usage in industry as well [2]. Most of these models have been developed in the frequency domain. This is very satisfactory for modeling the amplification of only one or a few simultaneous frequencies, for narrow-bandwidth TWTs, or for other stationary problems.

However, as the bandwidth of TWTs has increased, there has arisen more interest in simultaneously amplifying multiple frequencies. Nonlinear phenomena, such as intermodulation products and harmonic generation, have become significant, and codes have been modified or are being developed to model this phenomena [8]-[10]. When the number of frequencies is finite and of relatively small number $(<100)$, frequencydomain models are still applicable. However, if we extrapolate the use of TWTs to include not just concurrent multiple frequency operation, but the amplification of pulses in the TWT, then time-domain simulations may become necessary. Other applications of time-domain models include nonstationary problems such as modulated carrier amplification [11] or oscillation configurations [12].

Of interest to this paper are those technologies or techniques which rely upon pulse amplification, such as impulse radio, im-

Manuscript received August 29, 2003; revised February 18, 2004.

The authors are with the Department of Electrical and Computer Engineering, University of Wisconsin, Madison, WI 53706 USA (e-mail: converse@ cae.wisc.edu; booske@engr.wisc.edu; hagness@engr.wisc.edu).

Digital Object Identifier 10.1109/TPS.2004.828798 pulse radar, and experimental time-domain characterization of the TWT.

Ultrawideband (UWB) impulse radio is a technology utilizing extremely short pulses, on the order of $0.1-1.5 \mathrm{~ns}$ [13], [14]. Modulating this signal in time (i.e., varying pulse spacing) permits information to be transmitted. Potential benefits of impulse radio include increased spectral efficiency, secure communications, and immunity to multipath interference. This technology currently seems to be touted for short range or indoor communications that may not require the type of power regime that the TWT is meant for. However, it is possible that the technology will change or mature to be beneficial with longer range communications, and then TWTs may fill the power needs of these systems.

Impulse radar [15]-[17] is a technology that uses ultrashort pulses, such as a Gaussian pulse or one to three cycles of a sinusoidal signal, to obtain information about a target's presence, position, shape, or composition. The advantages of impulse radar are somewhat specific to the application, of which there are many. One of the biggest areas of interest for this technology is ground probing. Another application of emerging interest is "see-through-walls" surveillance radar. Because the impulse contains a wide bandwidth and has a short duration, it offers excellent range resolution and discrimination while propagating though solid materials. Other applications are defense oriented and involve overcoming stealth techniques which utilize absorptive coatings and preventing the target from becoming aware it is being scanned. Due to its wide bandwidth, impulse radar offers the intriguing possibility of being resistant to electronic countermeasures.

Time-domain characterization of the TWT involves the use of broadband pulses to measure dispersion, internal reflections, and other internal characteristics of a TWT in the presence of the beam. In particular, by using a broadband impulse to excite a system (in this case, a TWT), one can obtain information over a broad frequency range in a single experiment. For example, one could use impulse excitation as a quick and effective method to extract the small signal gain versus frequency characteristics of a TWT. Also, by using a short pulse, time-domain reflectometry (TDR) could be used to map internal reflections in space and in frequency. Since propagation characteristics are affected by the presence of the beam, an understanding of the nonlinear effects in the TWT response to impulse excitation would allow these measurements to be taken not only on the cold circuit, but also with the beam present.

A few investigations of pulse excitation of TWTs have been previously reported [18]-[32]. The majority of these studies 
utilized the TWT to generate a pulse, rather than to amplify an existing pulse. Also, most of these investigations were experimental. Published models for impulse amplification are scarce.

Several nonstationary TWT models have been previously developed and described in the literature [26], [33]-[35]. Each of these models has assumptions or features that might significantly limit their applicability for an investigation of impulse amplification. Such limitations include a lack of dispersion effects [33], inefficient incorporation of dispersion [34], and averaging over the period of a carrier wave [35]. The model most relevant to the study of impulse amplification in a TWT is reported in [26]. The model is based on a Green's function solution in the frequency domain, which is Fourier transformed into the time domain. Dispersion effects are added in the frequency domain. The process requires knowledge of quantities at future times. As these are not known, they are ignored. This introduces an artificial loss to the system. This loss effect has not been examined and its significance is unknown.

A few experiments have been conducted specifically to look at the effect of amplifying pulses [18], [32]. One experiment [32] appears to contradict the limitations of saturation, claiming a $2-W$ TWT produces a $20-\mathrm{W}$ output pulse. In single-frequency operation, a 2-W TWT would produce a maximum saturated output power of $2 \mathrm{~W}$. Although not explicitly stated, it would appear as though the calculated power is instantaneous peak power, whereas the $2-\mathrm{W}$ specification would represent an average steady-state power. Hence, it is unclear whether the discrepancy is a result of inappropriately comparing peak instantaneous power with average power or a result of unique physics when operating in pulsed mode.

In Section II of this paper, we present the development of a 1-D time-domain model which incorporates all the relevant physics of the TWT, without introducing any of the artificial effects found in other models. Section III presents an examination of the efficacy of pulse simulation using frequency-domain models. Section IV shows a favorable comparison of the time-domain model (as well as a frequency-domain model) with experimental measurements in the small and near-saturation regimes of a broadband TWT.

The model presented here and the observations made in this study are nominally applicable to all types of TWTs. However, the examples used and the experimental TWT which the model is compared against is a helix TWT, as this is the most interesting TWT for the study of very broadband signals.

\section{ONE-DimENSIONAL NONLINEAR NUMERICAL TWT MODEL-RUBEUS}

Our time-domain model, which we refer to as RUBEUS, combines a solution to the linear field equations with a nonlinear macroparticle beam model. The fields are solved using the telegrapher's equations (TEs) for a lossless transmission line. The TEs are a pair of coupled differential equations that describe the current $(i)$ and voltage $(v)$ on a transmission line using distributed capacitance $(C)$ and inductance $(L)$. The conventional form of the TEs for $z$-directed wave propagation are

$$
\frac{\partial v(z, t)}{\partial t}= \pm \frac{1}{C} \frac{\partial i(z, t)}{\partial z}
$$

and

$$
\frac{\partial i(z, t)}{\partial t}= \pm \frac{1}{L} \frac{\partial v(z, t)}{\partial z}
$$

Both the positive and negative equations are valid for the distributed circuit model. RUBEUS uses the negative equations.

The phase velocity and characteristic impedance of the wave traveling on such a line are

$$
V_{p}=\frac{1}{\sqrt{L C}} \quad Z_{0}=\sqrt{\frac{L}{C}} .
$$

Adopting the conventional approach of Pierce [1], the cold circuit helix phase velocity $v_{\mathrm{cc}}$ and the beam-wave interaction impedance $K$ are taken as substitutes for $v_{p}$ and $Z_{0}$, respectively. From $v_{\text {cc }}$ and $K$, an effective $L$ and $C$ can be determined for the TEs. In particular

$$
L=\frac{K}{v_{\mathrm{cc}}} \quad C=\frac{1}{v_{\mathrm{cc}} K} .
$$

These choices enable one to simulate a signal propagating along the helical waveguide. In the conventional forms of (1a) and (1b), $L$ and $C$ are constant real numbers. Therefore, all frequencies propagate with the same velocity and impedance. Consequently, use of this model to simulate a dispersive TWT is applicable to only one frequency at a time.

Since the helix TWT is dispersive and this dispersion is so important to the response of the device to pulsed operation, the frequency dependence of the impedance and capacitance must be added.

Because the TWT's dispersion is based upon lossless geometric effects, conventional 1-D techniques used to implement dispersion [36] cannot be used. In particular, dispersion specified as an explicit function of frequency cannot be used in a lossless time-domain model. From the same principle of causality with which the Kramers-Kronig relation is derived, it can be shown that it is not possible to realize an explicitly frequency-dependent phase velocity in a 1-D time-domain description without loss. On the other hand, dispersion specified as a function of wavenumber $\beta$ (inverse wavelength) can be used. This also has a physical appeal. Material dispersion results from relaxation responses of electrons, ion, or molecules, which are intrinsically temporal effects. In contrast, geometric (or waveguide) dispersion is associated with the wavelength of the wave and its relation to geometrical features such as wall dimensions or the distance of one turn of a helix. Dispersion data as a function of wavenumber can be determined from frequency-dependent dispersion data by the relation

$$
\beta=\frac{\omega}{v_{p}} .
$$

Recalling the relationships between phase (or cold circuit) velocity, interaction impedance, and the inductance and capaci- 


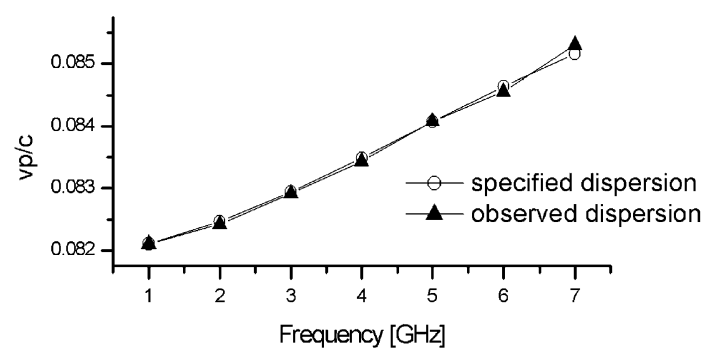

Fig. 1. Specified versus simulated phase velocity to validate dispersion method.

tance parameters in the TEs, the capacitance and inductance can be described as a function of wavenumber as follows:

$$
C(\beta)=\frac{1}{v_{p}(\beta) K(\beta)} \quad L(\beta)=\frac{K(\beta)}{v_{p}(\beta)} .
$$

Consistent with using wavenumber-dependent functions, it is appropriate to use a modification of the pseudospectral time-domain (PSTD) method [37] to numerically solve the transmission line equations. The PSTD method uses the fact that wave quantities vary as $e^{(j \omega t-j \beta z)}$. In the spatial frequency domain, the spatial derivatives of the transmission line voltage and current are $-j \beta V$ and $-j \beta I$, respectively. Therefore, to evaluate the spatial derivative of the current and voltage, one Fourier transforms the spatially dependent current or voltage, multiplies by $-j \beta$, then inverse Fourier transforms the quantities and updates the TEs. The modification to the method comes when one includes the wavenumber-dependent capacitance and inductance into the Fourier transform. This allows the dispersive effects to be included in the calculation. The numerical implementation of this method for the TEs is

$$
\begin{aligned}
V_{i}^{n} & =V_{i}^{n-1}+\Delta t F^{-1}\left[\frac{1}{C(\beta)} j \beta F\left(I^{n-1 / 2}\right)\right]_{i} \\
I_{i}^{n+1 / 2} & =I_{i}^{n-1 / 2}+\Delta t F^{-1}\left[\frac{1}{L(\beta)} j \beta F\left(V^{n}\right)\right]_{i} .
\end{aligned}
$$

$F$ and $F^{-1}$ represent forward and inverse Fourier transforms, respectively. Here second-order-accurate finite differences have been used to evaluate the time derivatives in (1a) and (1b).

Fig. 1 compares the specified or intended phase velocity for a simulation of a TWT with the actual phase velocity observed in a simulation based on (6) and (7). As can be seen from the figure, the agreement is very close. This illustrates that an intended dispersion can be implemented with good accuracy by the modified PSTD prescription of (6) and (7).

The above wave equations do not account for the presence of the electron beam moving along the axis or the effect of the beam on the wave. The beam's effect can be included by assuming that it is sufficiently close to the circuit to induce an image current $(I b)$ on the circuit [38]. This is incorporated into the PSTD equations by replacing the circuit current $I$ with $I+I b$. Of note is that in the above equations, the beam is coupled to the wave "capacitively." It is alternatively possible to mathematically couple the beam to the wave inductively or with a combination of inductive and capacitive coupling.
The effect of the wave on the beam and beam space charge effects are modeled with a macroparticle method [39]. The macroparticles are launched from one end of the simulation and propagated along the axis using the leapfrog method to determine position and velocity

$$
z^{n+(1 / 2)}=z^{n-(1 / 2)}+v^{n} \Delta t \quad v^{n+1}=v^{n}+a^{n+(1 / 2)} \Delta t
$$

where $x$ is position along the axis, $v$ is the velocity of the macroparticle, and $a$ is the acceleration of the macroparticle. The axial electric field component acting on the particles is calculated from the sum of the gradient of the line voltage and the gradient of the beam space charge potential times $R^{2}$

$$
E_{z}=-\left(\frac{\partial V}{\partial z}+R^{2} \frac{\partial}{\partial z}\right)
$$

where $R$ is the the plasma frequency reduction factor [39]. The space charge potential is calculated at the grid points from a grid sampled density, using Poisson's equation

$$
\nabla^{2} \varphi=-\frac{\rho}{\varepsilon_{0}}
$$

where $\rho$ is the grid-sampled density, which is determined similarly to the grid-sampled current. The beam, which consists of a stream of macroparticles, is mapped to the grid using a quadratic spline shape function [39].

In order to prevent instabilities and nonphysical forces on the particles, the force of the electric field on the particle must be weighted according to the same spline shape function. A discussion of shape functions and particle to grid weighting can be found in [39, Ch. 8].

The beam solution and the field solution combine to form the basis of the 1-D dispersive time-domain simulation of the TWT.

The model has been shown to implement dispersion with good accuracy, but a better test for the accuracy of the model is a comparison of single-frequency small signal gain with other established models and experimental results. This will be done in Section III, where reasonable agreement between RUEBEUS, an established frequency-domain model, and experimental results will be shown.

There are a few limitations to this time-domain model. First, because frequency (and, hence, wavelength)-dependent parameters are implemented in the spatial regime, these parameters cannot also, independently, vary as a function of $z$. So the phase velocity, interaction impedance, and plasma frequency reduction factor must be spatially uniform in RUBEUS. Attenuation, which in reality is frequency dependent, must be constant with wavelength in the model, as it is more important that it spatially vary, to account for the presence of a sever.

\section{COMParison With A FREQUenCy-Domain Model to SiMUlate PULSES}

Frequency-domain models work by simulating the response of the TWT to a discrete and finite number of pure harmonic excitations [2], [10]. In the nonlinear regime, they can account for harmonic and intermodulation effects as long as those frequencies are also included in the model. 
A finite-duration pulse in the time domain may be represented in the frequency domain by a continuum of frequencies with most of the power in a finite bandwidth. It is not immediately obvious that a finite number of frequencies can accurately represent the response of the TWT in the nonlinear regime. The frequency-domain model LATTE [10] was used to investigate the ability of frequency-domain models to simulate an inherently time-domain signal.

The first step was to determine which frequencies from the pulse would be used. Since an isolated pulse consists of an infinite number of frequencies, a finite subset must be chosen to be simulated in LATTE. If we assume that the time-domain pulse is not a single-incident pulse but a periodic series of widely separated pulses, then the frequency response is not a continuum of frequencies but an infinite series of discrete frequencies which are integer multiples of a base frequency (which is the frequency of repetition of the pulses). Since most of the energy is contained within a finite bandwidth, the pulse can be represented by a finite number of frequencies within this infinite series of discrete frequencies.

By keeping the maximum simulated frequency constant and by varying the repetition rate of the pulses in the pulse train, the number of frequencies used to simulate a pulse can be changed. A converged solution can then be found by increasing the number of frequencies until a convergence criterion is met. Note that this is not sufficient to determine the ability of a frequency-domain model to simulate the pulse response of an actual device. For that, a comparison with experimental results or a time-domain code is required.

To determine convergence of the frequency-domain model, we chose to use a Gaussian pulse as the test input to the model, varying the repetition rate to change the number of frequencies in the simulation. Convergence was determined from the following:

$$
C_{j}=\frac{\sum_{i=1}^{N_{0}}\left|x_{i, j}-x_{i, j-1}\right|}{\left|x_{i, j}\right|}
$$

where $C_{j}$ is the convergence value of the $j$ th iteration, $x_{i, j}$ is the $i$ th time point of the $j$ th iteration, and $N_{o}$ is the number of sample points in time.

Convergence was determined for signals in the linear and nonlinear regime. Here, "nonlinear" refers to the regime where there is a significant amount of power at frequencies outside the gain bandwidth of the TWT. If there are no harmonic or intermodulation (nonlinear) effects, then single and multifrequency studies indicate that the TWT would attenuate any frequencies outside the gain bandwidth. Therefore, if power at frequencies outside the gain bandwidth is significant at the end of the TWT, this could be considered a nonlinear regime. Figs. 2 and 3 show the converged output for an input Gaussian. Figs. 4 and 5 show the convergence value as a function of the number of frequencies.

As can be seen from Figs. 4 and 5, reasonable convergence, where we have chosen the point where $C_{j}^{*} 100$ becomes less than $0.5 \%$, for both cases seems to be obtained with 300 frequencies for a Gaussian pulse. For the remainder of this paper, the

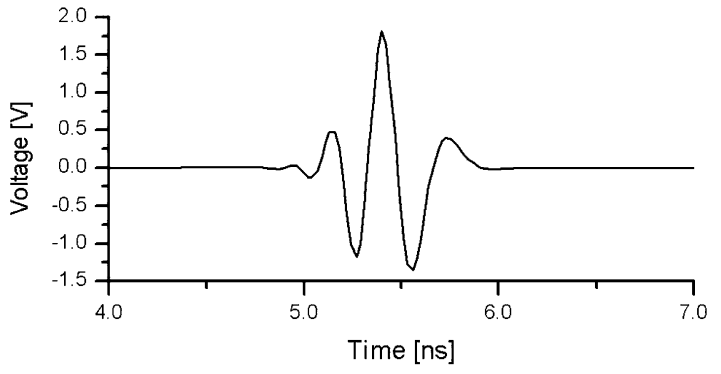

Fig. 2. Small signal output of frequency-domain model LATTE for input Gaussian.

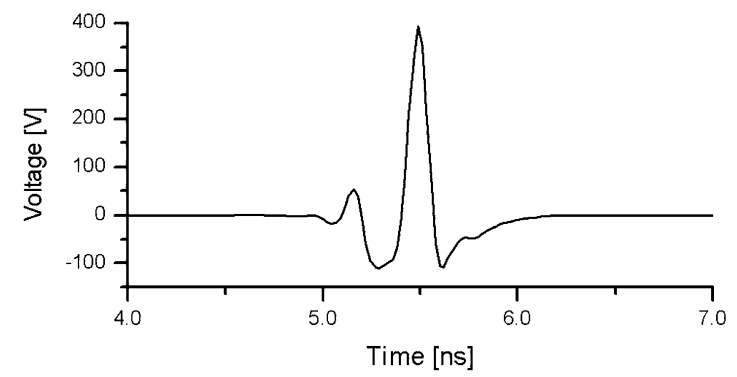

Fig. 3. Large signal output of frequency-domain model LATTE for input Gaussian.

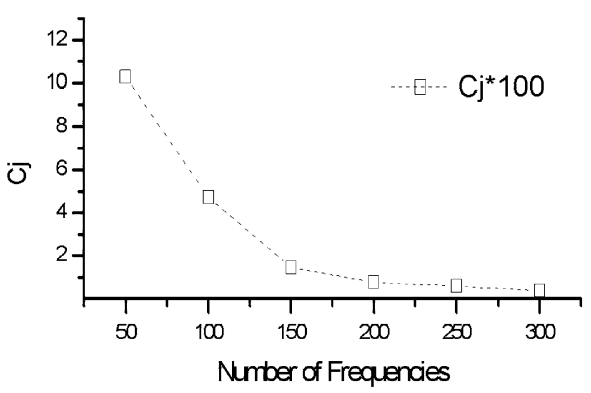

Fig. 4. Convergence of small signal response versus the number of frequencies.

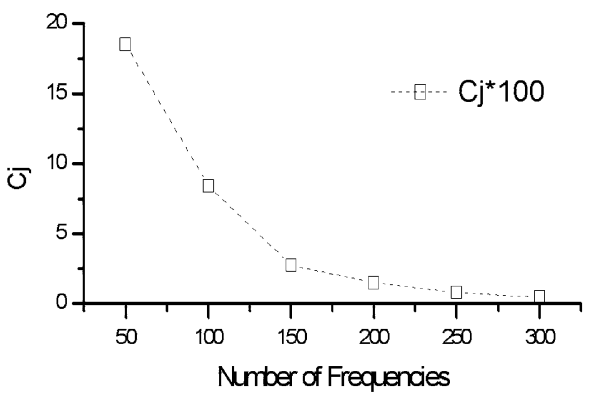

Fig. 5. Convergence of large signal response versus the number of frequencies.

number of frequencies used in LATTE will be 300. As can be seen, based upon the convergence criterion, this number of frequencies yields a converged solution.

\section{COMPARISON OF MODELS WITH EXPERIMENTAL MEASUREMENTS}

Experiments to validate models and further investigate pulsing phenomena were carried out on the experimental 


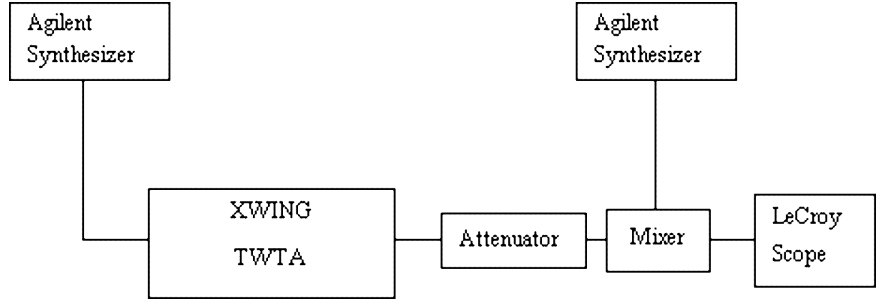

Fig. 6. Basic block diagram of experimental setup to perform small signal gain measurements.

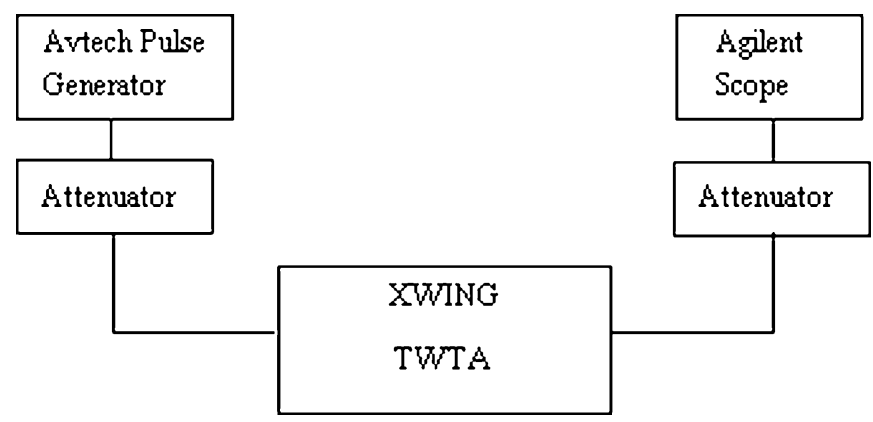

Fig. 7. Basic block diagram of experimental setup to measure amplified pulses using the XWING tube.

Wisconsin Northrop Grumman (XWING) TWT. This is a nominally $100-\mathrm{W}, 2-6-\mathrm{GHz}$ single-sever TWT (based on a production model) which was custom modified to allow greater diagnostic access to the fields and the beam for experimental research purposes [40]. Two measurement setups were used to measure the response of the XWING TWT. For singlefrequency small signal gain measurements, the setup was as follows. The single-frequency signal was input into the XWING TWT from a synthesizer, while the output was fed into a mixer and mixed down to $20 \mathrm{MHz}$. This allowed the amplitude of the output signal to be measured instantaneously (not requiring multiple pulses to capture all information). The impulse signal measurement setup was as follows. The output of a pulse generator was input into the XWING TWT, while the TWT output was input into an Agilent 86100 A wide-bandwidth oscilloscope. Pulse amplitude was controlled by adjusting the input attenuator. Due to heating concerns, the beam was repetitively pulsed at low-duty cycle, and data was acquired by accumulating the response to multiple pulses. Schematics for the two measurements are shown in Figs. 6 and 7.

Fig. 8 compares the small signal gain response of the two models and the experimental TWT. As can be seen from the figure, the time-domain model response fit is fairly good but not perfect. The reason for this is currently speculated to be related to 3-D effects. Both the models used in this study are 1-D models. Normally, 1-D models are sufficient to simulate the majority of interesting phenomena in a TWT or to act as an initial foray into a new investigation. The XWING TWT is an unusual device in that it was designed specifically for experimental investigations, and as a consequence of modifications adopted to allow greater diagnostic ability, control of the beam optics is not as precise as typical production models. Thus, nonaxial movement (e.g., beam ripple) of the electrons due to imperfect

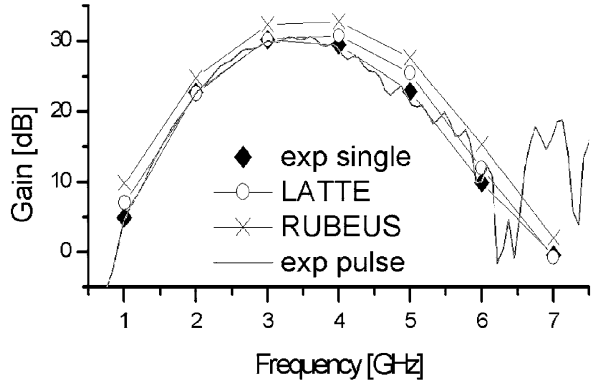

Fig. 8. Small signal gain versus frequency response of XWING (both single-frequency determined and pulse determined), RUBEUS, and LATTE.

TABLE I

DEVICE PARAMETERS USED IN SiMUlation

\begin{tabular}{|c|c|c|c|c|c|}
\hline & $v p / c$ & K [ohms] & $u_{o}[\mathrm{~m} / \mathrm{s}]$ & $\begin{array}{l}\text { I }_{\text {beam }} \\
\text { [Amps] }\end{array}$ & $\mathbf{r}_{\text {beam }}[\mathrm{cm}]$ \\
\hline 1 & 0.0821035 & 142.531 & $3.056 \mathrm{e} 7$ & 0.2 & 0.11 \\
\hline 2 & 0.0824704 & 111.81 & $3.056 \mathrm{e} 7$ & 0.2 & 0.11 \\
\hline 3 & 0.0829391 & 78.9001 & $3.056 \mathrm{e} 7$ & 0.2 & 0.11 \\
\hline 4 & 0.0834889 & 53.0666 & $3.056 \mathrm{e} 7$ & 0.2 & 0.11 \\
\hline 5 & 0.084076 & 35.2038 & $3.056 \mathrm{e} 7$ & 0.2 & 0.11 \\
\hline 6 & 0.0846463 & 23.001 & $3.056 \mathrm{e} 7$ & 0.2 & 0.11 \\
\hline 7 & 0.085154 & 14.5218 & $3.056 \mathrm{e} 7$ & 0.2 & 0.11 \\
\hline
\end{tabular}

magnetic optics may not be precisely characterized. Extensive work was done to try to obtain the best fit of the predicted gain from the models to the experimental data. To achieve a reasonable fit, the beam radius had to be increased from the measured and specified radius of about $0.5 \mathrm{~mm}$ to an effective value of $1.1 \mathrm{~mm}$. This increased radius and the possibility for non-1-D effects suggests that 2-D or 3-D effects are not completely ignorable in the XWING TWT. However, the fit of the small signal model to the experiment, in Fig. 8, appears to indicate that the 1-D approximation with the increased beam radius is sufficient to model the XWING tube. Table I gives details of the physical model used in the simulations including the fitted parameters. Details of the physical model for frequencies outside the operating regime of the TWT and how they were derived can be found in [41].

As can also be seen from Fig. 8, the frequency-domain model estimates a slightly better fit to the experimental results, by about $2 \mathrm{~dB}$, across the bandwidth of the device.

The reason the frequency-domain model gives a better approximation to the experimental results is that the frequency-domain model was used to fit to the experimental small signal gain. The frequency-domain model was chosen to fit to the experimental results over the time-domain model, due to implementation differences in cold circuit phase velocity and plasma frequency reduction factor and is discussed below.

The frequency-domain model implements the phase velocity and plasma frequency reduction factor as a function of frequency, while the time-domain model implements these parameters as a function of wavelength. For single-frequency excitation, the frequency remains fixed for all values of the TWT operating parameters, whereas the wavelength changes. The wavelength of the signal on the TWT is dependent on the presence and operating parameters of the beam. This variation of wavelength as a function of beam parameters leads to a 

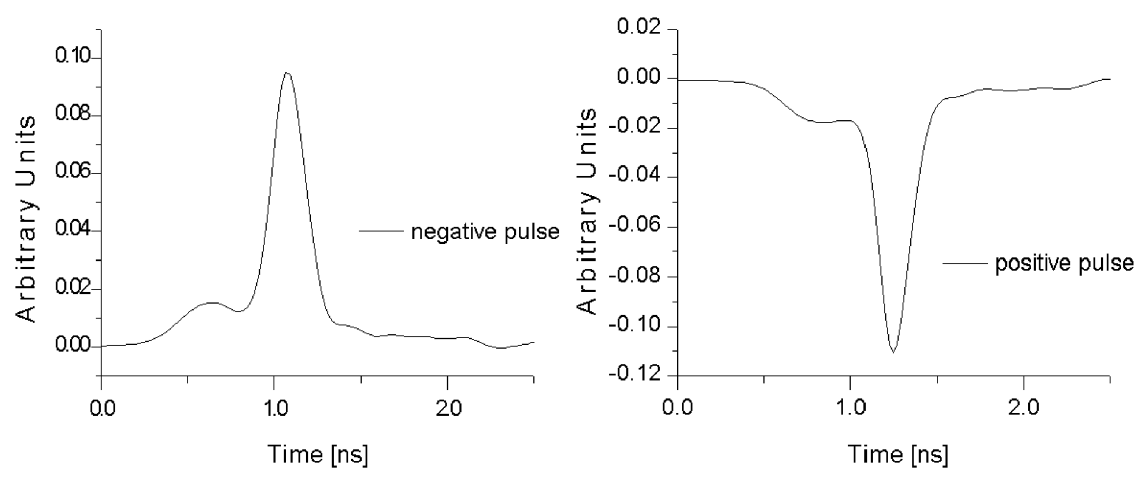

Fig. 9. Voltage versus time of positive and negative input signals used in experimental validation of models.
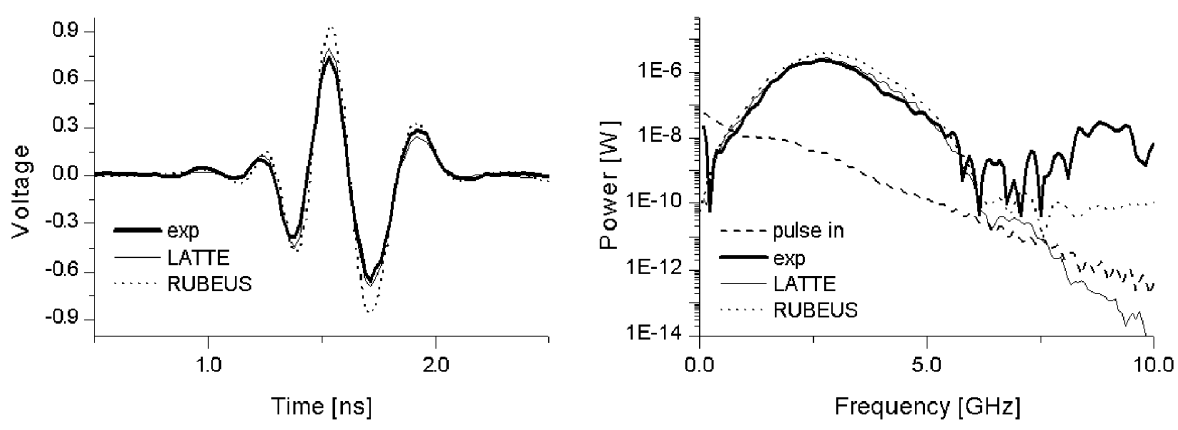

Fig. 10. Voltage versus time and power versus frequency for small signal positive input pulse.
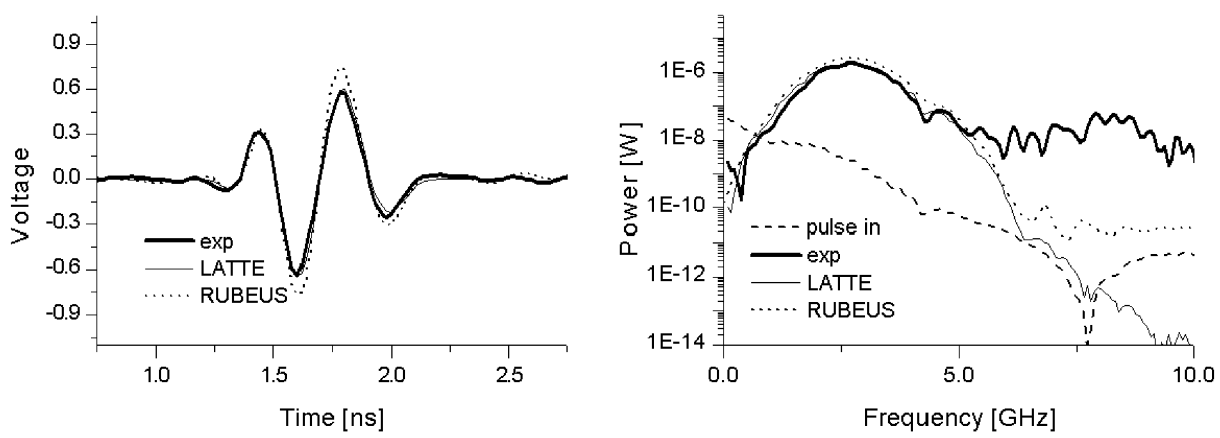

Fig. 11. Voltage versus time and power versus frequency for small signal negative input pulse.

discrepancy in the time-domain model between the specified and actual cold circuit phase velocity, making the estimation of cold circuit phase velocity more accurate in the frequency-domain mode. However, the plasma frequency reduction factor is better modeled as a function of wavelength than as a function of frequency, yielding a more accurate estimation of the plasma frequency reduction factor in the time-domain model. Since adjusting the radius of the beam affects the plasma frequency reduction factor and not the cold circuit phase velocity, it was decided that the frequency-domain model would be better suited to fit small signal gain model results to experimental results.

One other significant difference between the two models is related to the effect of attenuation on the phase velocity. LATTE's specified cold circuit phase velocity is constant regardless of the presence or magnitude of loss on the circuit. RUBEUS' specified cold circuit phase velocity is modified by the presence and amplitude of loss. In the low-loss region of the circuit (which constitutes much of the length of the TWT), this difference is negligible; however, in the sever region, this difference can be significant. These differences between the models lead to an approximately 2-dB difference in small signal gain across the gain bandwidth of the device.

Utilizing the fitted parameters from the small signal gain response comparison, the pulse responses of LATTE and RUBEUS are compared versus experimental measurements for a small and large, positive and negative input impulse. Fig. 9 shows the input signal waveforms. Figs. 10 and 11 compare the experimentally measured and the theoretically predicted output pulses for small signal positive and negative input polarities, respectively.

Figs. 12 and 13 compare the experimentally measured and the theoretically predicted output pulse for moderate signal positive and negative input polarities, respectively. The voltage and 

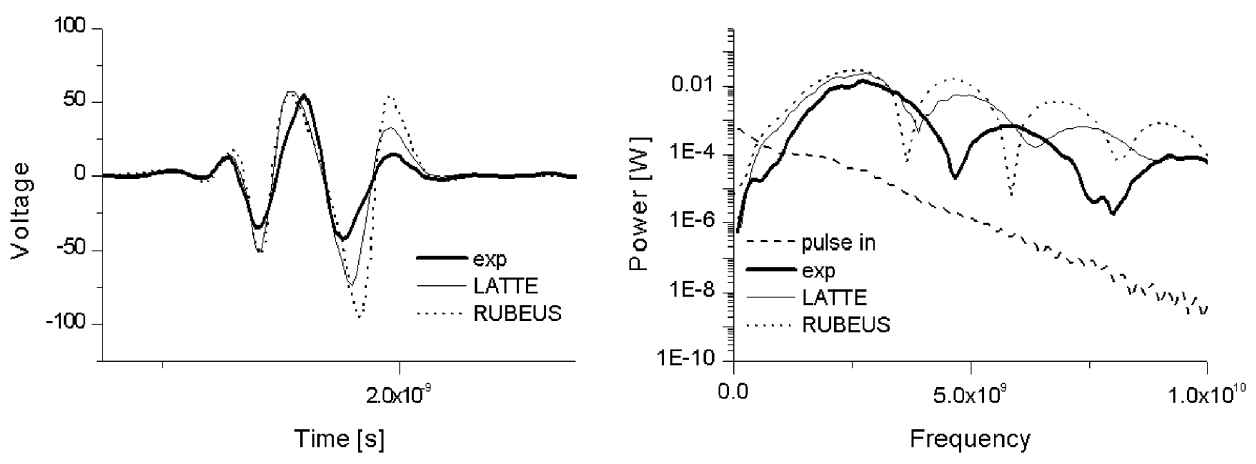

Fig. 12. Voltage versus time and power versus frequency for large signal positive input pulse.
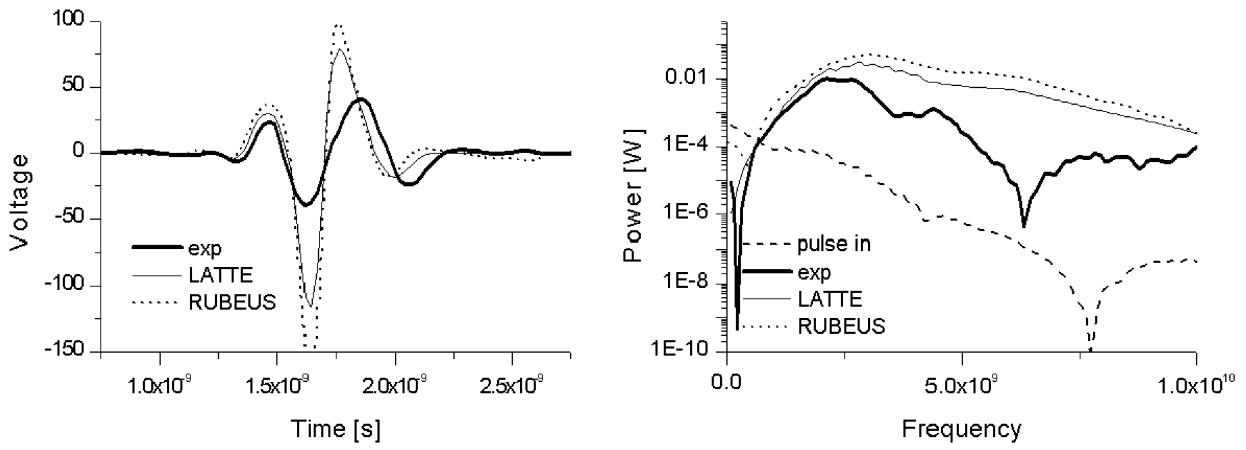

Fig. 13. Voltage versus time and power versus frequency for large signal negative input pulse.

power levels are much larger in Figs. 12 and 13 than in Figs. 10 and 11 . Note that in this case, "large" signal does not imply "saturated" signal.

From the small signal response of the positive and negative input pulse it can be seen that the models fit very well in the time domain, with RUBEUS being slightly larger in magnitude. This is consistent with the difference in small signal gain between LATTE and RUBEUS. In the frequency domain, the figures show a difference in the two models and the experiment above approximately $6 \mathrm{GHz}$. In this region, the experimental results show significantly more power than predicted by the models. This is most probably due to the measurement technique. The measurement of the output pulse of the XWING TWT was taken over a number of pulses to allow the oscilloscope to capture the signal. Any shot-to-shot fluctuations would be accumulated and could introduce high frequency noise. The expected experimental output in this region is three to four orders of magnitude lower than the power at the peak power frequencies (around 3-4 GHz), so noise above $7 \mathrm{GHz}$ could obscure the actual signal at those frequencies.

Larger signal response of the models compare well with each other, but not as well with the experiment. In this larger signal regime, 3-D effects may come into play. For example, with larger signals, beam bunching (and the electron repulsion force) is greater. Consequently, the beam radius may expand in this region. Bunch radius expansion effects are not included in either of the 1-D models. Simulations have indicated that as the beam radius increases from 0.5 to $1.1 \mathrm{~mm}$, the shape of the small signal gain response changes, favoring lower frequencies. If the beam radius increases at some point along the axis of the experi- mental device due to strong electron bunching, one might expect to see less growth at higher frequencies than the models predict (recall that the models maintain a constant radius under all conditions). This overestimated growth at higher frequencies by the models is consistent with the large signal results seen in Figs. 12 and 13. However, even with these differences, the agreement between the models and experiment is still considered to be good. The shapes in the time domain are approximately the same, and the models capture the characteristic "dips" in the frequency domain seen in the output of the positive large signal input pulse case of Fig. 12. Again observe that the large signal case, although showing evidence of nonlinear effects, is not saturated. In part due to limitations with the pulse generator, the saturated signal response was beyond the scope of this paper.

One final observation is that the good agreement between the predictions of the two models indicates that the frequency-domain model is able to accurately simulate a pulse through a TWT utilizing a large but finite number of frequencies. Thus, a time-domain model is not necessary if a frequency-domain model is available with the capacity to simultaneously calculate several hundred or more frequency components.

\section{Feasibility of Using Impulses to Measure Small SignAL GAIN}

By comparing the input and output of the small signal impulse in the frequency domain, the small signal gain as a function of frequency can be determined. The experimental small signal gain determined from a positive polarity input pulse is compared to the models and the small signal experimental gain 
from single-frequency measurements in Fig. 6. As seen from the figure, the agreement is very good. This experimentally validates that the small signal gain response of a TWT may be determined from the frequency response of a single broadband input pulse. There is some disagreement between the experimental results near $7 \mathrm{GHz}$. This, however, may be explained by shot-to-shot variations. Measurement of the output of the TWT was taken over many shots for the impulse case but determined by one shot for the single-frequency case. By using recently available high-digitizing-speed oscilloscopes, the impulse measurement could probably be repeated with a single shot.

\section{SUMMARY}

In this paper, we have introduced a new 1-D nonlinear time-domain code utilizing a novel method of implementing the effects of waveguide dispersion in one dimension in the time domain. It has been compared with an established frequency-domain model and experimental results. Both models have been used to favorably compare with the impulse response of an experimental TWT. Not only does this demonstrate the ability of the time-domain code to model the experimental impulse response, but it demonstrates that a frequency-domain model can also be used, provided it has the ability to simultaneously simulate several hundred or more frequency components. Also, the ability to characterize the small signal gain of the TWT using impulses has been experimentally confirmed.

\section{REFERENCES}

[1] J. R. Pierce, Traveling Wave Tubes, ser. Bell Laboratory Series. Princeton, NJ: Van Nostrand, 1950.

[2] T. Antonsen, Jr., and B. Levush, "CHRISTINE: A multifrequency parametric simulation code for traveling wave tube amplifiers," Naval Res. Lab., Washington, DC, Rep. NRL/FR/6840-97-9845, 1997.

[3] S. K. Datta, P. K. Jain, M. D. Raj Narayan, and B. N. Basu, "Nonlinear Eulerian hydrodynamical analysis of helix traveling-wave tubes for harmonic generation and its control," IEEE Trans. Electron Devices, vol. 46, pp. 420-425, Feb. 1999

[4] V. Srivastava and S. N. Joshi, "One-dimensional nonlinear model for helix TWTs," IETE Tech. Rev., vol. 6, no. 6, pp. 500-507, 1989.

[5] L. Kumar, R. S. Raju, S. N. Joshi, and B. N. Basu, "Modeling of a vane-loaded helical slow-wave structure for broad-band traveling-wave tubes," IEEE Trans. Electron Devices, vol. 36, pp. 1991-1999, Sept. 1989.

[6] C. L. Kory, "Fully three-dimensional helical RF field effects on TWT interaction," in Proc. IEEE 2nd Int. Vacuum Electronics Conf. (IVEC), 2001, pp. $163-168$.

[7] D. Chernin, T. Antonsen, Jr., B. Levush, and D. R. Whaley, "A threedimensional multifrequency large signal model for helix traveling wave tubes," IEEE Trans. Electron Devices, vol. 48, pp. 3-11, Jan. 2001.

[8] S. K. Datta, P. K. Jain, and B. N. Basu, "Control of IM3 distortion in helix TWT's by harmonic injection-An Eulerian hydrodynamical study," IEEE Trans. Electron Devices, vol. 48, pp. 62-66, Jan. 2001.

[9] R. G. Carter, B. Wolfgang, S. Vishnu, and G. Giuliano, "Computer simulation of inter-modulation distortion in broad-band, multi-carrier, travelling wave tube amplifiers," ITG-Fachbericht, no. 150, pp. 343-348, 1998.

[10] J. G. Wohlbier, J. H. Booske, and I. Dobson, "The multifrequency spectral Eulerian (MUSE) model of a traveling wave tube," IEEE Trans. Plasma Sci., vol. 30, pp. 1063-1075, June 2002.

[11] C. L. Kory and M. Andro, "Intersymbol interference investigations using a 3-D time-dependent traveling wave tube model," IEEE Trans. Plasma Sci., vol. 30, pp. 267-273, Feb. 2002.

[12] S. Bhattacharjee, J. H. Booske, C. L. Kory, D. W. van der Weide, S. Limbach, S. Gallagher, J. Welter, M. R. Lopez, R. M. Gilgenbach, R. L. Ives, M. E. Read, R. Divan, and D. C. Mancini, "Folded waveguide traveling wave tube sources for THz radiation," in IEEE Conf. Rec. Abstracts 30th Int. Conf. Plasma Science 2003, p. 189.
[13] M. Z. Win and R. A. Scholtz, "Impulse radio: How it works," IEEE Commun. Lett., vol. 2, pp. 36-38, Feb. 1998.

[14] — , "Ultra-wide bandwidth time-hopping spread-spectrum impulse radio for wireless multiple-access communications," IEEE Trans. Commun., vol. 48, pp. 679-691, Apr. 2000.

[15] M. G. M. Hussain, "Ultra-Wwideband impulse radar-An overview of the principles," IEEE Aerosp. Electron. Syst. Mag., vol. 13, pp. 9-14, Sept. 1998

[16] D. L. Black, "An overview of impulse radar phenomenon," IEEE Aerosp. Electron. Syst. Mag., vol. 7, pp. 6-11, Dec. 1992.

[17] D. Daniels, "Applications of impulse radar technology," in Proc. Radar Systems (Radar 97), Conf. Pub. No. 449, pp. 667-672.

[18] J. K. Pulfer and B. G. Whitford, "A simple method of generating nanosecond pulses at X band," Proc. IRE, vol. 49, pp. 968-968, May 1961.

[19] A. C. Beck and G. D. Mandeville, "Microwave traveling-wave tube millimicrosecond pulse generators," IRE Trans.-Microwave Theory Tech. vol. MTT-3, no. 6, pp. 48-51, Dec. 1955.

[20] D. P. Sobolev and V. E. Semenov, "Apparatus to measure low-noise signal levels in the presence of a periodic sequence of powerful radio pulses," Instrum. Exp. Tech., vol. 22, no. 2, pp. 499-502, Mar. 1979.

[21] W. A. Klute, "Pulse generation and shaping at microwave frequencies," Bell Lab. Rec., vol. 29, no. 5, pp. 216-220, May 1951.

[22] B. A. Stryukov and A. V. Luk'yanchikov, "Nonstationary processes during video-pulse excitation of an O-type traveling-wave tube," Sov. J. Commun. Technol. Electron., vol. 31, no. 2, pp. 124-128, Mar. 1986.

[23] M. A. Sletten, D. B. Trizna, and J. P. Hansen, "Ultrawide-band radar observations of multipath propagation over the sea surface," IEEE Trans. Antennas Propagat., vol. 44, pp. 646-651, May 1996.

[24] A. F. Dietrich, "8- and 11-Gc nanosecond carrier pulses produced by harmonic generation," Proc. IRE, vol. 49, pp. 972-973, May 1961.

[25] K. Miyauchi, "Traveling-wave tube nanosecond pulse generator in 24-Gc region," IEEE Trans. Microwave Theory Tech., vol. MTT-11, pp. 3-17, Jan. 1963.

[26] A. I. Man'kin and V. G. Shkol'nikov, "On the theory of nonstationary and nonlinear TWT," Radio Eng. Electron. Phys., vol. 29, no. 9, pp. 79-87, 1981.

[27] I. A. Man'kin, "Waveguide excitation by nonstationary extraneous currents," Radio Eng. Electron. Phys., vol. 24, no. 4, pp. 16-23, 1979.

[28] A. G. Lazerson, I. A. Man'kin, and V. G. Shkol'nikov, "Theoretical analysis of the amplification of wideband pulses in a TWT," Radio Eng. Electron. Phys., vol. 27, no. 8, pp. 118-123, 1981.

[29] I. A. Man'kin and V. G. Shkol'nikov, "Nonstationary processes during the passage of a current pulse through a retarding system," Radio Eng. Electron. Phys., vol. 28, no. 5, pp. 136-139, 1983.

[30] — " "Amplification of short pulses in a TWT," Radiotekh. Electron., vol. 31, no. 5, pp. 111-115, May 1986

[31] A. V. Luk'yanchikov and V. G. Shkol'nikov, "Formation of ultrashort radio pulses in a traveling-wave tube with a drift space," Radiotekh. Electron., vol. 32, no. 11, pp. 140-145, Nov. 1987.

[32] B. A. Stryukov and P. V. Milyayev, "A picosecond radio-pulse reflectometer,” Telecommun. Radio Eng., vol. 38, no. 5, pp. 85-87, 1983.

[33] I. J. Morey and C. K. Birdsall, "Taveling-wave-tube simulation: The IBC code," IEEE Trans. Plasma Sci., vol. 18, pp. 482-489, June 1990.

[34] V. Srivastava and S. N. Joshi, "Improved nonlinear model for multisignal analysis of helix TWTs," IEE Proc. - H, vol. 139, no. 2, pp. 129-134, Apr. 1992.

[35] H. P. Freund and E. G. Zaidman, "Time-dependent simulation of helix traveling wave tubes," Phys. Plasmas, vol. 7, no. 12, pp. 5182-5194, Dec. 2000.

[36] A. Taflove and S. C. Hagness, Computational Electrodynamics: The Finite-Difference Time-Domain Method, 2nd ed. Norwood, MA: Artech House, 2000.

[37] Q. H. Liu, "The PSTD algorithm: A time-domain method requiring only two cells per wavelength," Microw. Opt. Technol. Lett., vol. 15, no. 3, pp. 158-165, June 1997.

[38] S. Ramo, "Currents induced by electron motion," Proc. IRE, vol. 27, pp. 584-585, Sept. 1939.

[39] C. K. Birdsall and A. B. Langdon, Plasma Physics Via Computer Simulation. New York: IOP, 1991.

[40] M. Wirth, "Experimental investigations of a custom-made, helical traveling wave tube amplifier," M.S. thesis, Univ. Wisconsin, Madison, 2002.

[41] M. Converse, "Investigation of the mechanisms of pulse amplification in helical traveling wave tubes," Ph.D. dissertation, Univ. Wisconsin, Madison, 2003. 


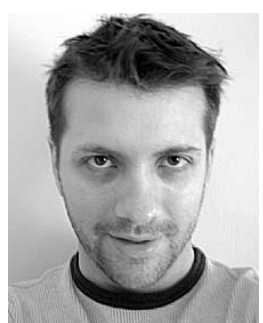

Mark C. Converse (S'91-M'96) received the B.S. degreee in electrical engineering and the M.S., and $\mathrm{Ph} . \mathrm{D}$. degrees from the University of Wisconsin, Madison, in 1996, 1999, and 2003, respectively.

During his graduate studies, he was engaged in plasma processing research involving damage evaluation/analysis and mitigation during the etching process. After obtaining his M.S. degree, he began research in microwave vacuum electronics investigating the impulse response of the helix traveling-wave tube. He is currently a Postdoctoral Scholar, University of Wisconsin, working on projects involving the microwave detection and treatment of breast and liver cancer using microwaves. His research interests include electromagnetic interactions with materials, electrical/biological interfaces, and organic electronics.

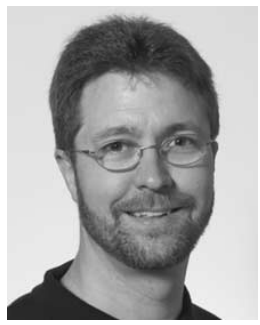

John H. Booske (S'82-M'85-SM'93) received the $\mathrm{Ph} . \mathrm{D}$. degree in nuclear engineering from the University of Michigan, Ann Arbor, in 1985.

From 1985 to 1989 , he was a Research Scientist at the University of Maryland, College Park. In 1990, he joined the faculty of the University of Wisconsin, Madison, where he is a Professor of Electrical and Computer Engineering. In 2001, he was appointed Director of the University of Wisconsin's Materials Science Program. His research interests include the experimental and theoretical study of coherent electromagnetic radiation sources and their applications, with emphasis in the RF, microwave, millimeter-wave, and terahertz regimes. Recent research activities include vacuum electronics, microwave and RF heating of materials, biological/biomedical applications of microwave and RF fields, high current density field emission cathodes, and ultrashallow junction fabrication in silicon CMOS transistors.

Prof. Booske received the University of Wisconsin Vilas Associate Award for research excellence and the U.S. National Science Foundation Presidential Young Investigator Award. He has been honored with many teaching awards, including the University of Wisconsin Chancellor's Distinguished Teaching Award and the Benjamin Smith Reynolds Award for Excellence in Teaching Engineering. He served as Codirector of a U.S. Department of Defense MURI99 consortium on innovative microwave vacuum electronics and as Director of a MURI04 consortium on cathode field emission and RF window breakdown in high-power microwave sources.

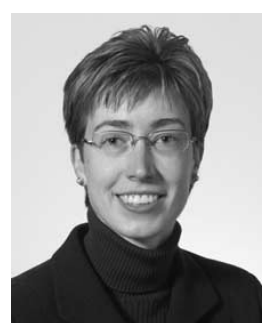

Susan C. Hagness (S'91-M'98) received the B.S. degree with highest honors and the Ph.D. degree in electrical engineering from Northwestern University, Evanston, IL, in 1993 and 1998, respectively. While working toward the $\mathrm{Ph} . \mathrm{D}$. degree, she was a National Science Foundation (NSF) Graduate Fellow and a Tau Beta Phi Spencer Fellow.

Since 1998, she has been with the University of Wisconsin, Madison, where she is currently an Associate Professor in the Department of Electrical and Computer Engineering and a Faculty Affiliate of the Department of Biomedical Engineering. Her research interests include finite-difference time-domain (FDTD) theory and applications, microwave imaging and sensing techniques for biological and medical applications, and full-wave analysis and design of photonic microstructures. She coauthored (with A. Taflove) Computational Electrodynamics: The Finite-Difference Time-Domain Method, 2nd. ed. (Norwood, MA: Artech House, 2000).

Dr. Hagness received the Presidential Early Career Award for Scientists and Engineers from the White House in 2000. She also received the Holdridge Excellence in Teaching Award from the Electrical and Computer Engineering Department, University of Wisconsin. In 2002, she received the Booker Fellowship Award from the United States National Committee of the International Union of Radio Science. She was also named one of the 100 top young innovators in science and engineering in the world by the Massachusetts Institute of Technology's Technology Review magazine. In 2003, she received the University of Wisconsin Emil Steiger Distinguished Teaching Award. She is currently serving as an Associate Editor for the IEEE ANTENNAS AND WIRELESS PROPAGATION LETTERS and as a Member of the IEEE Antennas and Propagation Society Administrative Committee. 